\title{
Tratamiento endovascular de pseudoaneurisma roto de aorta abdominal luego de litotricia extracorpórea
}

\author{
Endovascular aortic pseudoaneurism after extracorporeal lithotripsy
}

Luciano Chiaramonte' ${ }^{1}$, Hernán Acostaํ', Carmelo Bitonte'1, Lorena Villagra'

\section{RESUMEN}

Se describe un paciente de sexo masculino de 64 años de edad al que tres semanas previas se le realizó litotricia extracorpórea. Ingresó por dolor abdominal persistente y shock hipovolémico. La Tomografía demostró un hematoma retro peritoneal y un pseudoaneurisma de aorta abdominal. Se efectuó tratamiento endovascular , implantando dos stents balón-expandibles cubiertos con PTFE (Cheatham-Platinum Stent - Numed ${ }^{\circledR}$ ), en forma telescopada para asegurar la cobertura de la ruptura. El paciente requirió internación prolongada pero con una evolución favorable, sin nuevas intercurrencias. Conclusión: Las técnicas endovasculares nos brindan una alternativa eficaz y segura para el tratamiento del pseudo aneurisma roto.

Palabras claves: pseudoaneurisma de aorta abdominal, stent recubierto, litotricia extracorpórea.

\section{ABSTRACT}

A 64-year-old-man was admitted to the hospital with severe abdominal pain and hypovolemic shock, three weeks before the patient was treated with extracorporea shock wave lithotripsy. CT scan showed a retroperitoneal hematoma and a pseudo aneurysm of infrarenal abdominal aorta. An endovascular treatment was performed two balloon expandable stents, PTFE covered (Cheatham-Platinum Stent - Numed ${ }^{\circledR}$ ), were telescopically implanted to assure complete coverage of the rupture.

The patient required prolonged hospital stay but its outcome was favorable without new Intercurrences. Conclusion: Endovascular repair is a safe and effective treatment for ruptured abdominal aortic pseudo aneurysm.

Key words: aortic pseudo-aneurysm, stent graft, extracorporeal lithotripsy.

Revista Argentina de Cardioangiología Intervencionista 2014;5(3):221-224

\section{INTRODUCCIÓN}

La ruptura de aorta abdominal está generalmente vinculada al aneurisma aterosclerótico, siendo el riesgo de esta más frecuente cuanto mayor es el diámetro y depende en alguna medida también de su morfología, ya que los aneurismas saculares tienen una tendencia a la ruptura más precoz que los fusiformes .

Los pseudoaneurismas tienen etiología diferente de la ateroesclerosis, relacionándose habitualmente al trauma, ya sea este penetrante o cerrado y afectan estructuras vasculares habitualmente normales. Otras etiologías son las infecciones peri vasculares de órganos digestivos y las complicaciones de la cirugía reparadora de la aorta abdominal.

Es una entidad de evolución catastrófica ya que la mortalidad luego de la ruptura es de aproximadamente un

\footnotetext{
1. Sanatorio Pasteur. San Fernando del Valle de Catamarca, Argentina.

$\triangle$ Correspondencia: Luciano Chiaramonte. Maipú 960, (4700) San Fernando del valle de Catamarca |lucianochiara@hotmail.com | Teléfono: 0383-154223993.
}

Conflictos de intereses: no existen.

Recibido: 26-5-2014 | Aceptado: 7-7-2014

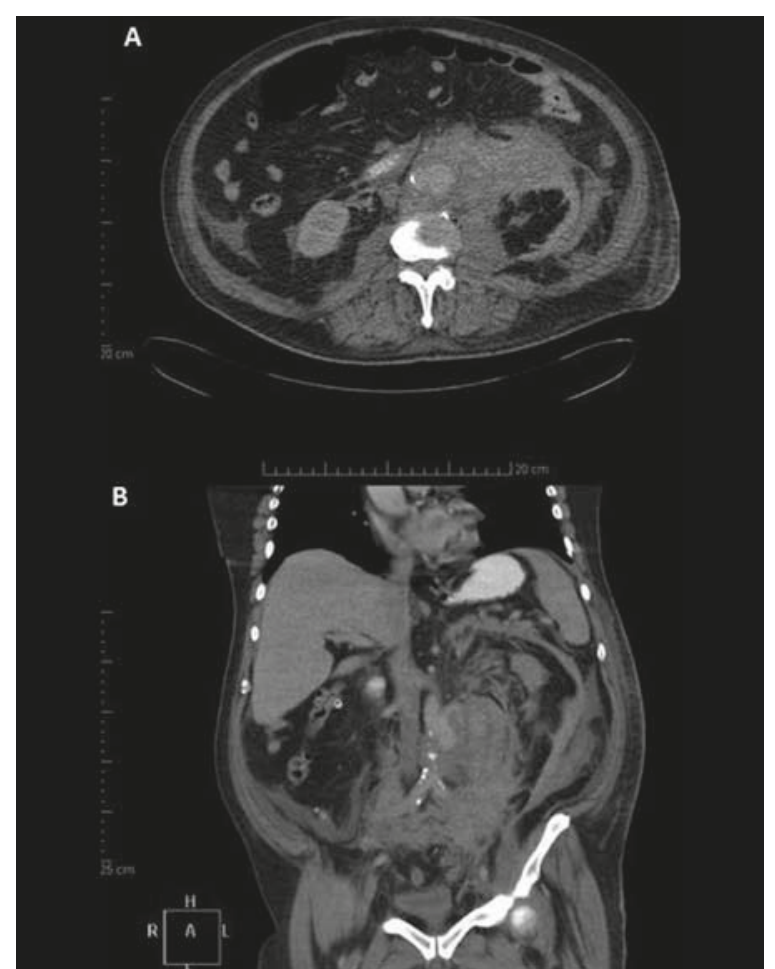

Figura 1. Tomografía de aorta abdominal. A. Corte axial: hematoma retro peritoneal y perirrenal izquierdo. B. Corte coronal: saco del pseudoaneurisma por debajo de la arteria renal izquierda. 


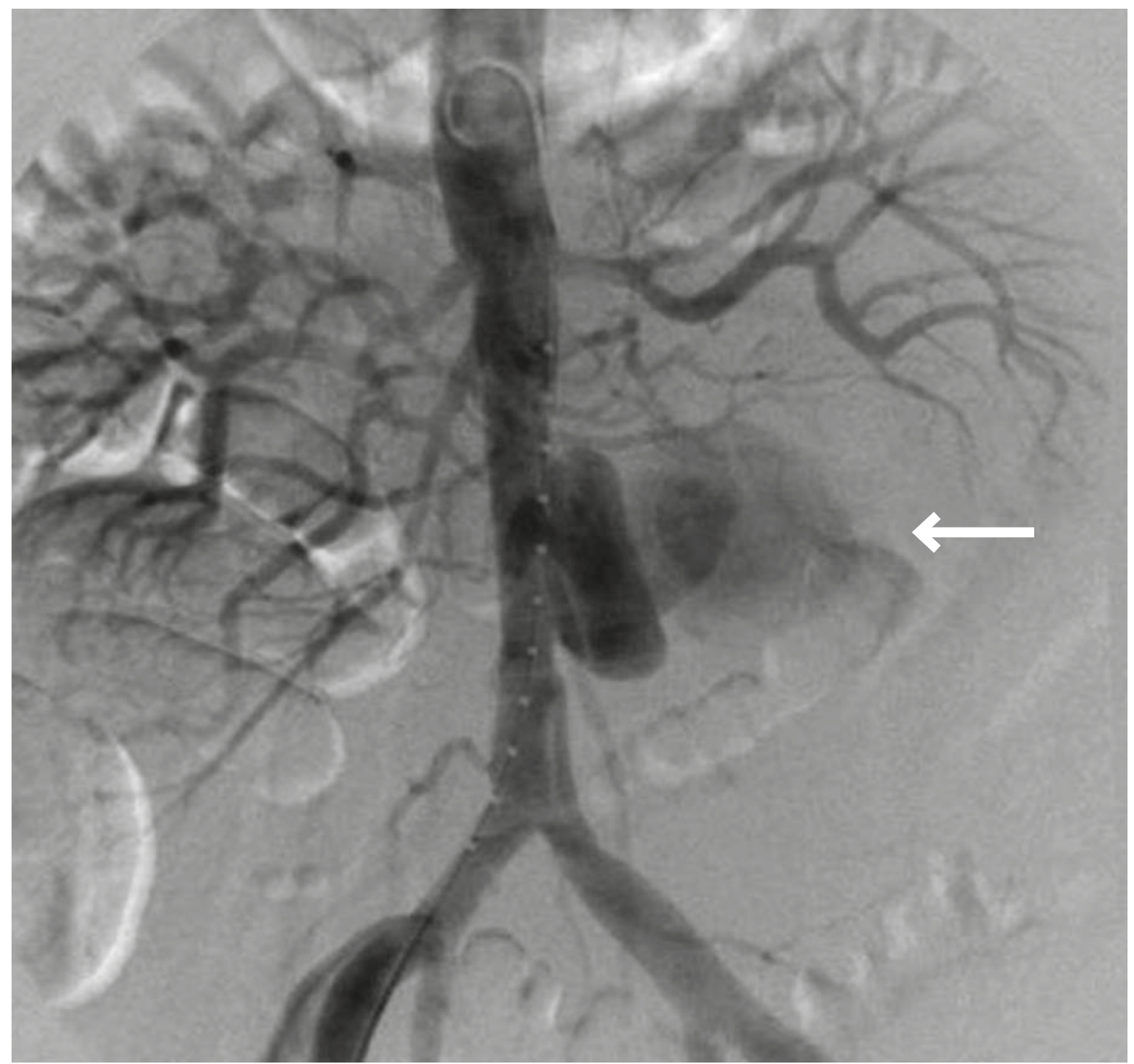

Figura 2. Aortograma abdominal: aorta de calibre normal; por debajo de la arteria renal izquierda se observa el saco bilobulado del pseudoaneurisma (flecha)

$80 \%$, un tercio de las cuales pueden producirse antes del arribo al hospital. ${ }^{1}$

\section{PRESENTACIÓN DEL CASO}

Paciente de sexo masculino de 64 años de edad con antecedentes de hipertensión arterial. Tres semanas previas a la consulta se le realizó una sesión de litotricia extracorpórea debido a litiasis pielocalicial del riñón derecho.

Ingresó a Unidad Coronaria por dolor abdominal persistente, al momento de la admisión presentaba parámetros de shock hipovolémico con hipotensión arterial y palidez generalizada, deterioro del sensorio y oliguria. Se realizó TC de Abdomen con contraste que mostró una aorta abdominal de calibre normal (diámetro $28 \mathrm{~mm}$ a nivel infra renal), un gran hematoma que comprometía retro peritoneo, la celda renal izquierda y el psoas homolateral. A $38 \mathrm{~mm}$ por debajo de la arteria renal izquierda presentaba una dilatación sacular compatible con un pseudo aneurisma que conec- taba con la luz vascular a través de un cuello angosto (Figura 1).

Se realizó aortograma abdominal, que mostró que el sitio de efracción se encontraba a $4.5 \mathrm{~cm}$ por debajo de la arteria renal izquierda y que la longitud de la misma era de $3,5 \mathrm{~cm}$ (Figura 2).

Dada la gravedad del cuadro se decidió su resolución endovascular. Se efectuó abordaje femoral derecho percutáneo con introductor 14Fr. y se implantó de un stent balón expandible de platino, cubierto con PTFE (Cheatham - Platinum Stent - Numed ${ }^{\circ}$ ) de $45 \mathrm{~mm}$ de longitud, montado sobre un balón de $24 \mathrm{~mm}$ de diámetro en el segmento más proximal de la lesión, finalmente para asegurar la correcta cobertura del cuello del pseudoaneurisma se implantó en forma telescópica un segundo stent de iguales dimensiones en el segmento inferior. En el aortograma de control se constató el correcta aposición con adecuado sellado de la fuga de material de contraste (Figura 3).

El paciente requirió una internación prolongada (14 


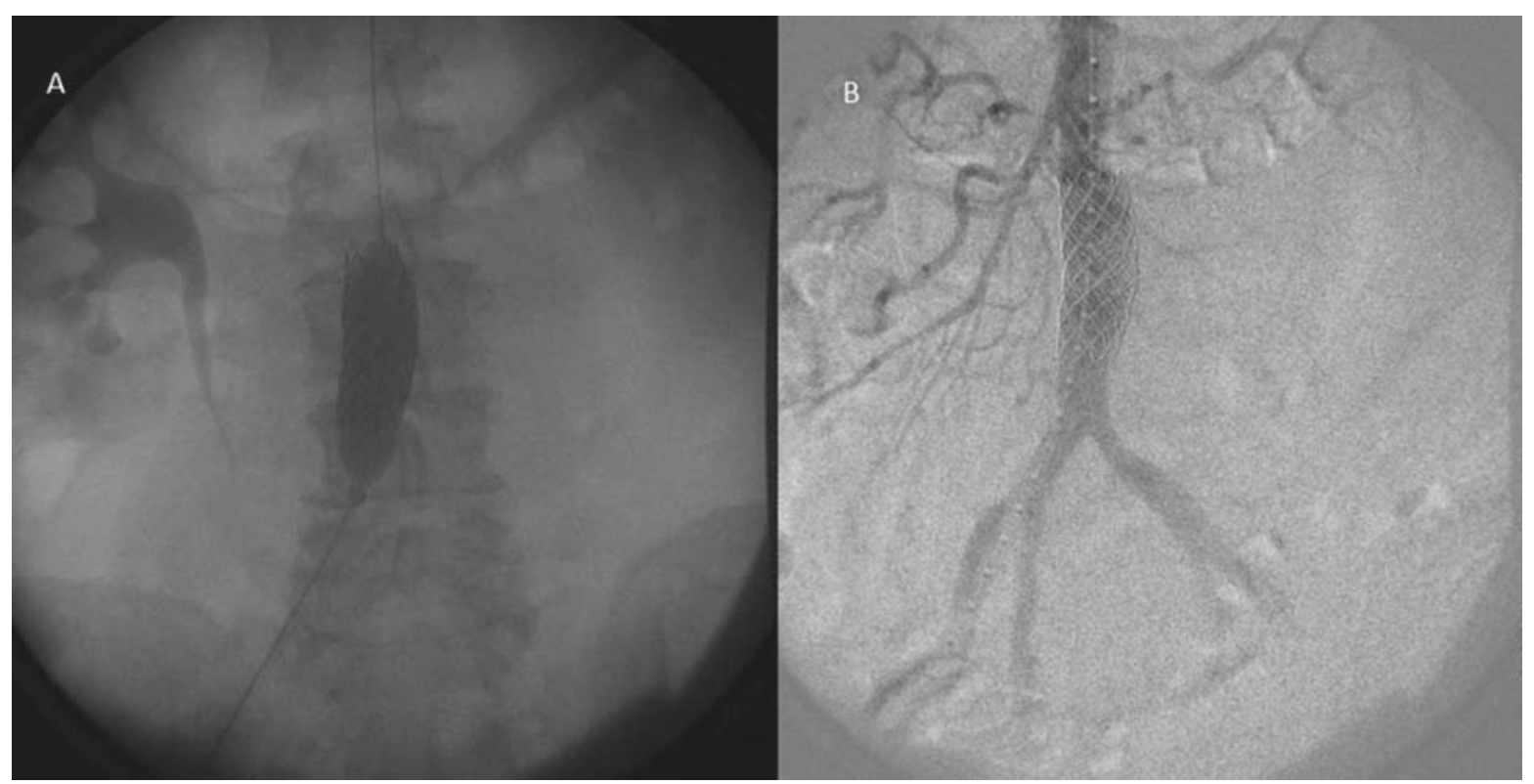

Figura 3. A. Implante del stent sobre balón de $24 \mathrm{~mm}$. B. Angiograma de control que muestra la ausencia de contraste en el exterior de la prótesis.

días) para recuperarse de las complicaciones vinculadas al sangrado masivo pero sin nuevas intercurrencias. Un eco Doppler de aorta abdominal realizado a las 48 hs del procedimiento mostró ausencia de flujo en el exterior de las prótesis.

En el seguimiento a tres meses mantuvo una evolución favorable, se mantuvo asintomático, una nueva Ecografía de control mostró señal normal en aorta y ambas ilíacas, sin evidencia de flujo en el saco del pseudoaneurisma (Figura 4).

\section{DISCUSIÓN}

Existen reportes de que pacientes con aneurisma de aorta abdominal, severa calcificación de la pared o ambos hallazgos simultáneamente, pueden presentar la ruptura de la aorta abdominal en forma tardía (entre 3 y 12 semanas), luego de la práctica de litotricia extracorpórea. ${ }^{2,3}$ En el presente caso se observó que la complicación se desarrolló en un paciente cuya aorta no aparentaba sufrir ninguna de las condiciones antes mencionadas.

El registro IMPROVE ${ }^{(4)}$ que comparó en forma randomizada, los resultados entre la cirugía convencional y el tratamiento endovascular en pacientes con aneurisma de aorta abdominal roto, reporta una mortalidad a 30 días similar entre ambas estrategias (37.4\%, vs $35.4 \% \mathrm{P}$ $=0.73)$, con reducción de la estadía en unidades críticas (6.3 vs 4.2 días) y hospitalaria (12.2 vs 9.8 días) con el tratamiento endovascular.

Existen publicaciones sobre el uso de prótesis expandibles por balón en el tratamiento de las lesiones traumáticas de la aorta fundamentalmente en la edad pediátri$\mathrm{ca}^{(5)}$, pero la mayoría de los reportes de casos en la edad adulta se refieren al uso de prótesis auto expandibles. ${ }^{(6)}$ Una limitación de estas nuevas técnicas es la dificultad para el acceso inmediato a este tipo de dispositivos, debi-

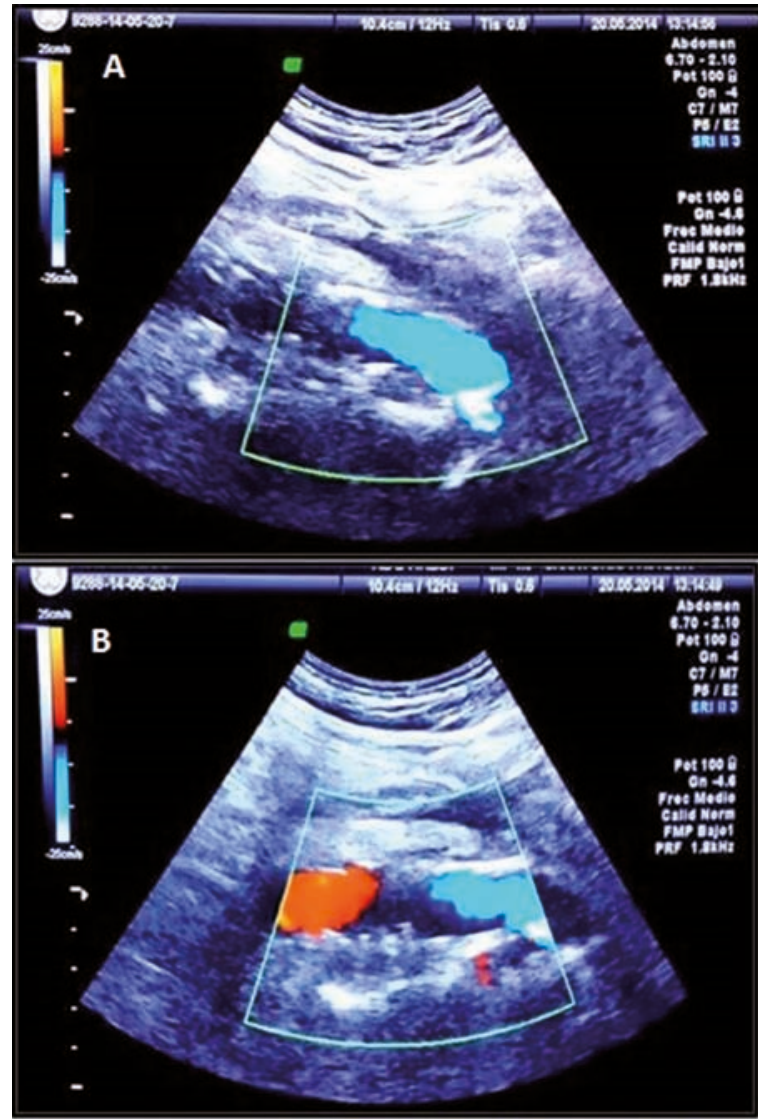

Figura 4. Eco Doppler de aorta abdominal. Corte longitudinal de aorta a nivel infrarrenal que evidencia diámetros y velocidades normales hasta la bifurcación, sin flujo a través de la prótesis.

do a su alto costo, diversidad de medidas y modelos, además de la escasa posibilidad de stock institucional. Dada la escasa ventana de tiempo que otorga la gravedad de estos cuadros, hacen que se deba utilizar la prótesis que se encuentra disponible y no aquella que se considera como la más recomendable. 


\section{CONCLUSIÓN}

Si bien el abordaje quirúrgico es el tratamiento convencional, el desarrollo de las técnicas endovasculares pro-

\section{BIBLIOGRAFÍA}

1. Reimerink J. Systematic review and meta-analysis of population-based mortality from ruptured abdominal aortic aneurysm. Br J Surg 2013;100 (11):1405e13.

2. Neri, E. Localized dissection and delayed rupture of the abdominal aorta after extracorporeal shock wave lithotripsy. J Vasc Surg 2000;31:1052-5.

3. G.Gugulakis. Rupture of the abdominal aorta following extracorporeal shock-wave Lithotripsy. Eur J Sur 1998; 164:233-235.

4. Endovascularoropen repairstrategy for ruptured abdominal aortic aneurysm: 30 day outcomes from IMPROVE randomised trial. BMJ 2014; 348:f7661. ducida en las últimas décadas nos brinda una alternativa eficaz y segura para resolver este tipo de cuadros de gravedad extrema y muy alto riesgo operatorio con el tratamiento habitual.

5. Goldstein BH. Percutaneous balloon-expandable covered stent implantation for treatment of traumatic aortic injury in children and adolescents Am J Cardiol 2012 Nov 15;110(10):1541-5.

6. Tshomba Y, On-label use of commercially-available abdominal endografts for para-anastomotic aneurysms and pseudoaneurysms after infrarenal abdominal aortic aneurysm open repair. Eur J Vasc Endovasc Surg. 2013 Dec;46(6):657-66. 